\title{
Methylated Alteration of SHP1 Complements Mutation of JAK2 Tyrosine Kinase in Patients with Myeloproliferative Neoplasm
}

\author{
Jun-Jun Yang' ${ }^{1 \&}$, Hui Chen ${ }^{1 \&}$, Xiao-Qun Zheng ${ }^{1}$, Hai-Ying Li² ${ }^{2}$ Jian-Bo Wu², Li- \\ Yuan Tang ${ }^{3}$, Shen-Meng Gao ${ }^{2 *}$
}

\begin{abstract}
SHP1 negatively regulates the Janus kinase 2/signal transducer and activator of transcription (JAK2/STAT) signaling pathway, which is constitutively activated in myeloproliferative neoplasms (MPNs) and leukemia. Promoter hypermethylation resulting in epigenetic inactivation of SHP1 has been reported in myelomas, leukemias and other cancers. However, whether SHP1 hypermethylation occurs in MPNs, especially in Chinese patients, has remained unclear. Here, we report that aberrant hypermethylation of SHP1 was observed in several leukemic cell lines and bone marrow mononuclear cells from MPN patients. About 51 of $118(43.2 \%)$ MPN patients including 23 of $50(46 \%)$ polycythaemia vera patients, 20 of $50(40 \%)$ essential thrombocythaemia and 8 of $18(44.4 \%)$ idiopathic myelofibrosis showed hypermethylation by methylation-specific polymerase chain reaction. However, SHP1 methylation was not measured in 20 healthy volunteers. Hypermethylation of SHP1 was found in MPN patients with both positive $(34 / 81,42 \%)$ and negative $(17 / 37,45.9 \%)$ JAK2V617F mutation. The levels of SHP1 mRNA were significantly lower in hypermethylated samples than unmethylated samples, suggesting SHP1 may be epigenetically inactivated in MPN patients. Furthermore, treatment with 5-aza-2'-deoxycytidine (AZA) in K562 cells showing hypermethylation of SHP1 led to progressive demethylation of SHP1, with consequently increased reexpression of SHP1. Meanwhile, phosphorylated JAK2 and STAT3 were progressively reduced. Finally, AZA increased the expression of SHP1 in primary MPN cells with hypermethylation of SHP1. Therefore, our data suggest that epigenetic inactivation of SHP1 contributes to the constitutive activation of JAK2/STAT signaling. Restoration of SHP1 expression by AZA may contribute to clinical treatment for MPN patients.
\end{abstract}

Keywords: SHP1 - myeloproliferative neoplasm patients- hypermethylation

Asian Pac J Cancer Prev, 16 (6), 2219-2225

\section{Introduction}

Philadelphia-negative myeloproliferative neoplasms (MPNs), mainly including polycythaemia vera (PV), essential thrombocythaemia (ET) and idiopathic myelofibrosis (IMF), represent a wide range of clonal hematological stem cell malignancies(Tefferi and Vardiman, 2008). Constitutive activation of Janus kinase $2 /$ signal transducers and activators of transcription (JAK2/STAT) signaling caused by JAK2V617F mutation is considered to be the major pathogenesis of MPNs (James et al., 2005; Levine et al., 2005). The JAK2V617F mutation in Exon 14 has been observed in almost $98 \%$ of patients with PV and 50-60\% of patients with ET and IMF. Other mutations such as JAK2 mutation in Exon 12(Pietra et al., 2008), thrombopoietin receptor (MPL515) mutation (Pardanani et al., 2006) and mutation in the inhibitory adaptor protein LNK (Oh et al., 2010) also result in the aberrant activation of JAK2/STAT signaling in JAK2V617F mutation-negative MPNs. Therefore, agents aiming to inhibit JAK2 activity such as Ruxolitinib (Verstovsek et al., 2012b), TG101348 (Pardanani et al., 2011) and CYT387 (Tyner et al., 2010) had been entered into clinical trials. Also, Ruxolitinib had been approved by US Food and Drug Administration (FDA) for treating intermediate- and high-risk IMF.

SHP1, a member of the SHP family of proteins, is located on chromosome 12p13 and encodes a protein of nonreceptor type protein-tyrosine phosphatase(Shen et al., 1991). SHP1 contains two tandem Src homology (SH2) domains, a catalytic domain, and a $\mathrm{COOH}$-terminal tail. SHP1 is known to be an important negative regulator through interaction with multiple signaling molecules including Epo-R, IL-2R, CD22 and B-cell receptor in various signal transduction pathways (Zhang et al., 2000). The SHP1 gene is comprised of 17 Exons and is activated 


\section{Jun-Jun Yang et al}

from two different promoters. The proximal promoter P2 initiating gene transcription from exon 2 (alternatively known as exon 1b), is always utilized by the hematopoietic cells. DNA methylation within promoter P2 of the SHP-1 gene had been reported to result in a strong reduction of SHP-1 mRNA and protein in leukemia, lymphoma, and HTLV-1-transformed T cells (Oka et al., 2002; Nakase et al., 2009). Several proteins including NF-KB p65, PU.1 and SP1 regulate the expression of SHP-1 through interaction with SHP-1 promoter P2 (Wlodarski et al., 2007).

Three families of proteins including the protein inhibitors of activated STATs (PIAS), the suppressors of cytokine signaling (SOCS), and SHP has been reported to negatively regulate JAK2/STAT signaling (Benekli et al., 2003). Although emerging data obtained in vitro and in animal models have indicated that JAK2V617F mutation may be sufficient for the development of MPNs especially PV, there is still evidence suggest that additional molecular alterations may contribute to the development of both JAK2V617F-negative and JAK2V617F-positive MPNs. Loss of SHP1 expression by hypermethylation in SHP1 promoter region rather than due to inactivating mutations had been associated with aberrant JAK2/STAT activation in multiple myeloma (Chim et al., 2004), leukemia (Oka et al., 2002), malignant T-cell lymphoma (Zhang et al., 2005) and advanced stages of cutaneous T-cell lymphoma (Witkiewicz et al., 2007). Therefore, epigenetic inactivation of negative regulator of JAK2/ STAT signaling may contribute to the activation of JAK2/ STAT signaling in addition to JAK2V617F mutation(Jost et al., 2007). However, whether SHP1 is hypermethylated in MPN patients especially in Chinese MPN patients still remains unclear.

The aim of this study was to investigate the epigenetic inactivation of SHP1 in MPNs. We found aberrant hypermethylation of SHP1 was observed in bone marrow mononuclear cells from MPN patients. Loss of SHP1 expression by aberrant hypermethylation failed to negatively suppress JAK2/STAT signaling. AZA restored the expression of SHP1 in K562 cells and primary MPN cells with hypermethylation of SHP1 but not in HEL cells with unmethylation of SHP1. Restoration of SHP1 expression finally resulted in the inhibition of JAK2/STAT signaling. Thus, restoration of SHP1 expression by AZA in MPN patients may contribute to the clinical treatment combined with routine therapy.

\section{Materials and Methods}

\section{Cell lines and bone marrow mononuclear cells from MPN patients}

K562, HEL, U937, U266, Raji, Jurkat and NB4 cell lines were cultured in RPMI 1640 supplemented with $10 \%$ heat-inactivated fetal bovine serum (Invitrogen, Carlsbad, CA, USA) in humidified $37^{\circ} \mathrm{C}$ incubator with $5 \% \mathrm{CO}_{2}$ for the present study. Bone marrow mononuclear cells were obtained from MPNs patients (Supplemental Table 1-3) including 50 patients with PV, 50 with IMF, and 18 with ET. Informed consents in accordance with the Declaration of Helsinki were given by this patients and all manipulations were approved by the Medical Science Ethic Committee of Wenzhou Medical University. Bone marrow mononuclear cells were isolated by Ficoll density gradient centrifugation (GE Healthcare, Uppsala, Sweden) and were cultured in RPMI 1640 supplemented with $10 \%$ fetal bovine serum (Invitrogen) in humidified $37{ }^{\circ} \mathrm{C}$ incubator with $5 \% \mathrm{CO}_{2}$. AZA (Sigma-Aldrich, St Louis, MO, USA) was dissolved in distilled water and kept at $-20^{\circ} \mathrm{C}$ until used. Final concentrations of AZA in this study were 5 and $10 \mu \mathrm{M}$.

\section{Methylation-specific polymerase chain reaction (MSP)}

High-molecular-weight genomic DNA was isolated by standard protocols (Qiagen, Valencia, CA, USA) from diagnostic bone marrow mononuclear cells and leukemic cell lines. Treatment of DNA with bisulfite was performed by a commercially available kit (Millipore, Billerica, MA, USA). The methylation-specific polymerase chain reaction (MSP) for promoter P2 was designed by MethPrimer software (Li and Dahiya, 2002). Primers for methylation of SHP1 were F: 5'-TTT TTT TTG TTG TGT TTT AAA ACG A-3' and R: 5'-CTC AAA TAC AAC TCC CAA TAC CG-3'. Primers for the unmethylation of SHP1 were F: 5'-TTT TTT TGT TGT GTT TTA AAA TGA-3' and R: 5'-CTC AAA TAC AAC TCC CAA TAC CAA-3'.

\section{DNA sequencing}

The identity of the methylated and unmethylated sequences was confirmed by automated DNA sequencing. PCR products were gel purified, sequenced bidirectionally, and analyzed on an automated DNA sequence analyzer by commercial company (Geneskybiotech, Shanghai, China).

\section{JAK2V617F mutation analysis}

JAK2V617F mutation status was assessed by direct DNA sequencing (Geneskybiotech) on total bone marrow genomic DNA. Primers for sequencing were F: 5'- GGT TTC CTC AGA ACG TTG A; R: 5'-CAT TGC TTT CCT TTT TCA CAA.

\section{mRNA extract and quantitative real-time PCR}

Total mRNA was extracted by TRIzol (Invitrogen, Carlsbad, CA, USA) following the manufacture's protocol. Quantitative real-time polymerase chain reaction (qRT-PCR) analysis for SHP1 was performed by SYBR Green PCR Master Mix (Applied Biosystems, Foster City, CA). Relative expression was calculated using the $2^{-\Delta \Delta C T}$ method. Primer sequences of SHP1 were F: 5' -TTT CAA GAA GAC GGG GAT TG-3'; R: 5'-CGG ACT CCT GCT TCT TGT TC-3'. Primer sequences of GAPDH were F: 5'GGT CGG AGT CAA CGG ATT TG-3'; R: 5'-ATG AGC CCC AGC CTT CTC CAT-3'. GAPDH housekeeping gene was used for normalization.

\section{Western blotting}

For whole protein extracts, leukemic cells were washed with PBS, harvested and resuspended in RIPA lysis buffer (50 mM TrisHCl, $150 \mathrm{mM} \mathrm{NaCl}, 0.1 \%$ SDS, $0.5 \%$ sodiumdeoxycholate, $1 \%$ NP-40, 1 mM PMSF, and $2 \mathrm{mg} / \mathrm{mL}$ aprotinin, $100 \mathrm{mM}$ leupeptin, $\mathrm{pH} 8.0$ ) and separated on an $12 \%$ SDS-polyacrylamide gel and 
transferred to nitrocellulose membranes. After blocking with $5 \%$ nonfat milk, the membranes were incubated with an appropriate dilution of the primary antibody, followed by incubation with the horseradish peroxidase (HRP)conjugated secondary antibody (Cell Signal Technology, Boston, USA). The following antibodies were used: SHP1 (Abcam, Cambridge, MA, USA); JAK2, p-JAK2 ${ }^{\text {Tyr1007, }}$ STAT3 and $p$-STAT ${ }^{\text {tyr705 }}$ (Cell Signal Technology). The signals were detected by chemiluminescence phototopeHRP kit (Cell Signaling, Danvers, MA, USA). Blots were stripped and reprobed with anti- $\beta$-actin antibody (Cell Signal Technology) as an internal control. All experiments were repeated three times.

\section{Statistical analysis}

The significance of the difference between groups was determined by Student's t-test. A P value of less than .05 was considered statistically significant. All statistical analyses were performed with SPSS software (Chicago, IL, USA).

\section{Results}

The aberrant hypermethylation of SHP1 is observed in leukemic cell lines and bone marrow mononuclear cells from MPN patients

To assess the methylation status of SHP1 promoter in leukemia, we firstly detected the SHP1 methylation by the methylation-specific PCR in 7 leukemic cell lines. As indicated in Figure 1A, K562 and U266 showed complete methylation, while U937, Raji and Jurkat were hemizygous methylation. However, NB4 and HEL cells were totally unmethylated. Next, status of SHP1 methylation was detected in $118 \mathrm{MPN}$ patients including

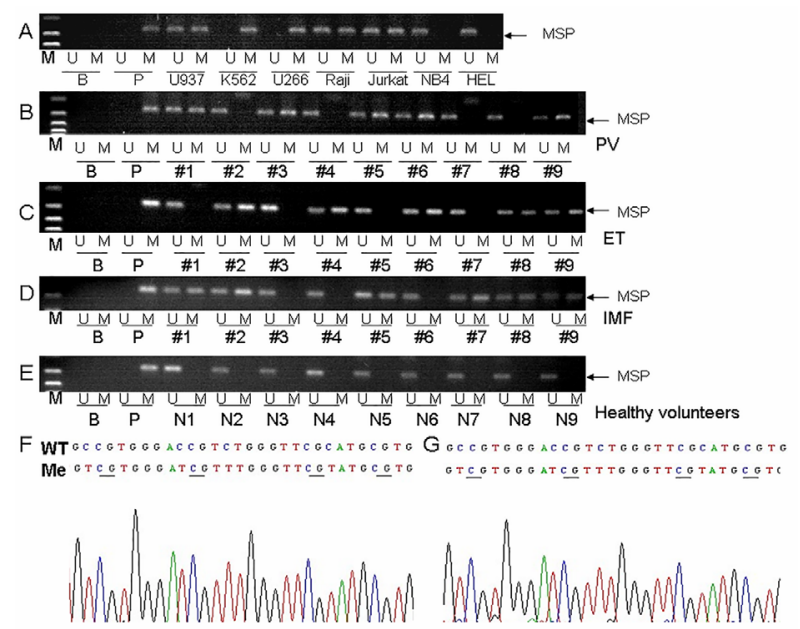

Figure 1. Methylation-specific Polymerase Chain Reaction (MSP) for SHP1. (A) Representative M-MSP and U-MSP for 7 leukemic and lymphoma cell lines including U937, K562, U266, Raji, Jurket, NB4, and HEL. B: Blank. P: positive control of methylated DNA. (B-D) Representative MMSP and UMSP analysis for 118 patients with MPN including 50 patients with PV (B), 50 with ET (C), and 18 with IMF (D). (E) Representative MMSP and UMSP for 20 healthy volunteers. (F and G) DNA sequencing of SHP-1 in untreated K562 cells (WT) and bisulfite-converted K562 cells (Me). Methylated cytosine residues in $\mathrm{CpG}$ dinucleotide remained as $\mathrm{C}$, whereas unmethylated cytosine read as $\mathrm{T}$ after bisulfite conversion
Table 1. Analysis of CpG Island Methylation of SHP1 in Bone Marrow Mononuclear Cells from Patients with MPN

\begin{tabular}{lrr}
\hline Diagnosis & \multicolumn{2}{c}{ SHP1 methylation } \\
& + & - \\
\hline PV & $23 / 50(46 \%)$ & $27 / 50(54 \%)$ \\
ET & $20 / 50(40 \%)$ & $30 / 5(60 \%)$ \\
IMF & $8 / 18(44 \%)$ & $10 / 18(56 \%)$ \\
Total MPN & $51 / 118(43 \%)$ & $67 / 118(57 \%)$ \\
Healthy volunteers & $0 / 20(0 \%)$ & $20 / 20(100 \%)$ \\
\hline
\end{tabular}

50 patients with PV, 50 with ET, and 18 with IMF. As indicated in Table 1, 23 of 50 (46\%) PV patients were hypermethylated (Figure 1B), 20 of 50 (40\%) ET patients (Figure 1C) and 8 of 18 (44\%) IMF patients (Figure 1D) showed hypermethylation. Totally, 51/118 (43.2\%) MPN patients showed methylation (Table 1). However, SHP1 methylation was not measured in 20 healthy volunteers (Table 1 and Figure 1E). In order to further verify the reliability of M-MSP, unconverted and bisulfite-converted DNA from K562 and 10 MPN patients with positive M-MSP of SHP1 were performed sequencing. Our data indicated that sequences of $\mathrm{CpG}$ island in K562 (Figure 1F) cell line and MPN patients (Figure 1F) showed the expected nucleotide change.

The expression of SHP1 is downregulated in hypermethylated leukemic cell lines and MPN patients

To verify the association between aberrant methylation and gene expression in leukemic cell lines and MPN patients, we firstly detected the mRNA and protein levels of SHP1 in 7 leukemic cell lines. The mRNA and protein levels of SHP1 in K562 and U266 cells were significantly lower than those in U937, Raji and Jurkat cells with hemizygous methylation and NB4 and HEL cells with unmethylation (Figure 2A-B). The protein levels of SHP1 in K562 and U266 cell lines showing complete methylation were almost undetected, suggesting that methylation mediated the silence of SHP1. We then measured the mRNA levels of SHP1 in 118 MPN patients. According to the methylation status, all MPN patients were divided into two groups including unmethylated MPN patients and hypermethylated MPN patients. We found SHP1 mRNA levels in unmethylated MPN patients were 2.8-fold higher than that in methylated MPN patients (Figure 2C). Next, we compared SHP1 mRNA levels in healthy volunteers and MPN patients. As indicated in Figure 2D, SHP1 levels in healthy volunteers were about 1.5-fold higher than those in MPN patients. Moreover, we analyzed the SHP1 mRNA levels in patients with PV, ET and IMF. No obvious differences of SHP1 levels were found between PV, ET and IMF patients (Figure 2E).

In order to explore whether JAK2V617F mutation affects SHP1 methylation. We firstly checked the frequency of JAK2V617F mutation in our cohort. JAK2V617F mutation was detected in 81/118 $(68.6 \%)$ MPN patients including 44/50 (88\%) PV, 27/50 (54\%) ET, and 10/18 (55\%) IMF. Then SHP1 methylation was analyzed according to JAK2V617F mutation status in PV, ET and IMF patients. SHP1 methylation was detected in 
Jun-Jun Yang et al

20/44 (45.5\%) PV with positive JAK2V617F mutation, 9/27 (33.3\%) ET with positive JAK2V617F mutation, 5/10 (50\%) IMF with positive JAK2V617F mutation. In a word, SHP1 methylation was detected in 34/81 (42\%) MPN patients with JAK2V617F mutation compared with 17/37 (45.9\%) MPN patients without JAK2V617F mutation $(p>0.05)$. Finally, SHP1 levels were analyzed in MPN patients divided by JAK2V617F mutation. No significant difference of SHP1 mRNA level was found in MPN patients with JAK2V617F mutation compared with those without JAK2V617F mutation (Figure 2F).

\section{AZA inhibits JAK2/STAT signaling through restoring the expression of SHP1}

In order to explore the biological function of SHP1 methylation, K562 showing complete hypermethylation of SHP1 was used a model in vitro. As shown in Figure 3A, AZA treatment resulted into a progressive demethylation of SHP1 by M-MSP with decreasing amplification intensity and by positive U-MSP with increasing amplification intensity. The progressive SHP1 demethylation was correlated with a parallel reexpression of SHP1 mRNA and protein (Figure 3B-C). Consequently, this led to a corresponding reduction of phosphorylated JAK2 and STAT3 (Figure 3C). Interestingly, the levels of nonphosphorylated JAK2 and STAT3 were almost unchanged, suggesting that SHP1 reexpression interfered with phosphorylation of JAK2 and STAT3.

As our data had indicated that HEL cells indicated unmethylation of SHP1 (Figure 1A), we then determined whether AZA failed to modulate the expression of SHP1 in HEL cells. We found SHP1 mRNA and protein levels were not changed in AZA-treated HEL cells than untreated cells (Figure 3B-C). Also, AZA treatment did not affect the levels of phosphorylated JAK2 and STAT3 in HEL cells (Figure 3C). Therefore, the SHP1 may play an important role in the AZA-induced inhibition of JAK2/ STAT Signaling.

AZA increases the levels of SHP1 in primary MPN cells with SHPI hypermethylation, but fails to alter the expression of SHP1 in those with SHP1 unmethylation.

Finally, we determined whether AZA increased the levels of SHP1 in primary MPN cells through demethylation of SHP1. Bone marrow mononuclear cells from 40 MPN patients with SHP1 hypermethylation including $15 \mathrm{PV}, 15 \mathrm{ET}$, and $10 \mathrm{IMF}$ were treated with AZA for 4 days, and then the levels of SHP1 were measured as described. We found the expression of SHP1 were elevated in 11 of 15 PV (Figure 4A), 12 of 15 ET (Figure 4B), and 7 of $10 \mathrm{IMF}$ (Figure 4C). Also, bone marrow mononuclear cells from 40 MPN patients without SHP1 hypermethylation including 15 PV, 15 ET, and $10 \mathrm{IMF}$ were treated with AZA for 4 days. However, the expressions of SHP1 were not changed in those with SHP1 unmethylation (data not shown). MSP was analyzed in a patient with SHP1 hypermethylation. AZA treatment led to a progressive demethylation of SHP1 by U-MSP with increasing amplification intensity and M-MSP with decreasing amplification intensity (Figure 4D). Thus, these data suggest that AZA treatment increased the expression

2222 Asian Pacific Journal of Cancer Prevention, Vol 16, 2015
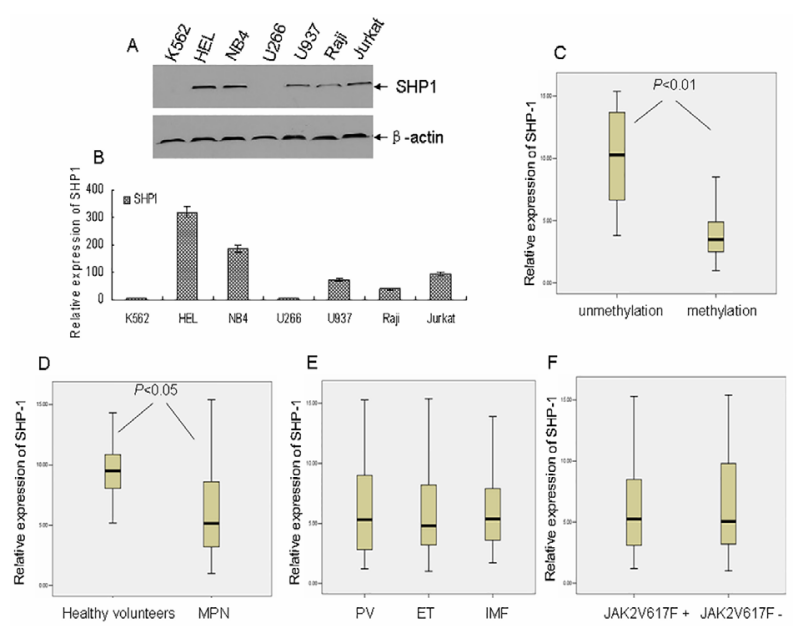

Figure 2. Expression Analysis of SHP1 in Leukemic and Lymphoma Cell Lines and 118 Patients with MPN. (A-B) The protein and mRNA levels of SHP1 in 7 leukemic and lymphoma were detected by Western blotting and qRT-PCR. (C) Relative expression of SHP1 in unmethylated and methylated patients with MPN. (D) The expression of SHP1 in 20 healthy volunteers and all MPN patients. (E) The expression of SHP1 in 50 patients with PV, 50 with ET, and 18 with IMF. (F) The 118 patients with MPN are divided into two groups according to JAK2V617F mutation. The levels of SHP1 are measured in MPN patients with JAK2V617F mutation and without JAK2V617F mutation, respectively

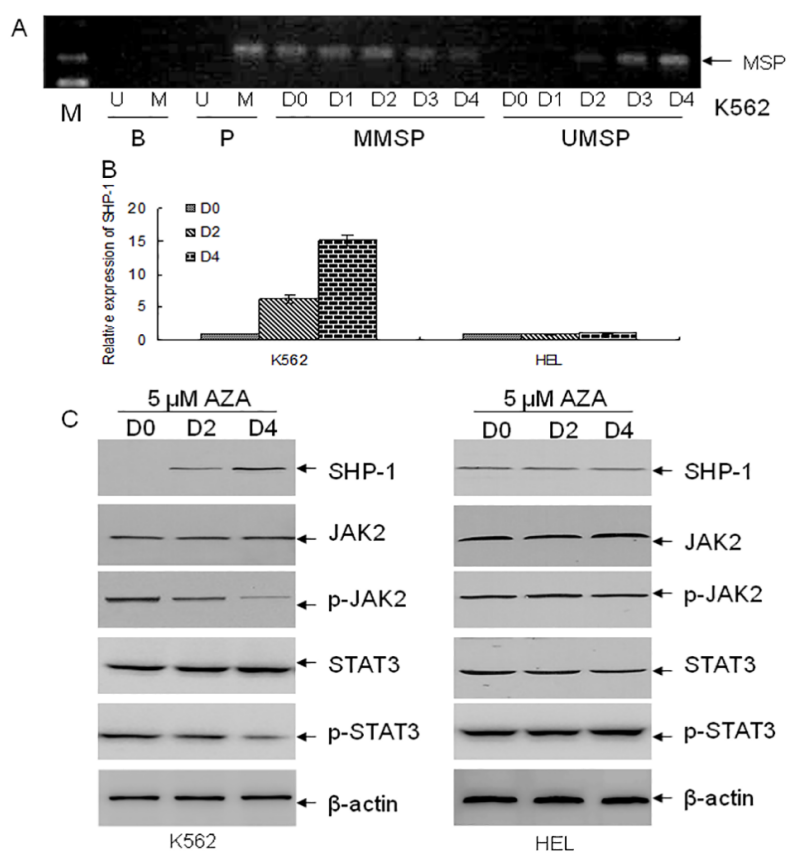

Figure 3. Restoration of SHP1 Inhibits JAK2/STAT Signaling in K562 Cells through AZA-induced Demethylation. (A). MMSP and UMSP were analyzed in K562 cells treated with $5 \mu \mathrm{M}$ AZA for indicated days. (B). SHP1 expression was measured by qRT-PCR in K562 and HEL treated with $5 \mu \mathrm{M}$ AZA for indicated days. (C). JAK2/STAT signaling was detected in K562 and HEL treated with $5 \mu \mathrm{M}$ AZA for indicated days

of SHP1 by reducing the methylation of SHP1 in primary MPN cells.

\section{Discussion}

In this study, we investigated the status of SHP1 


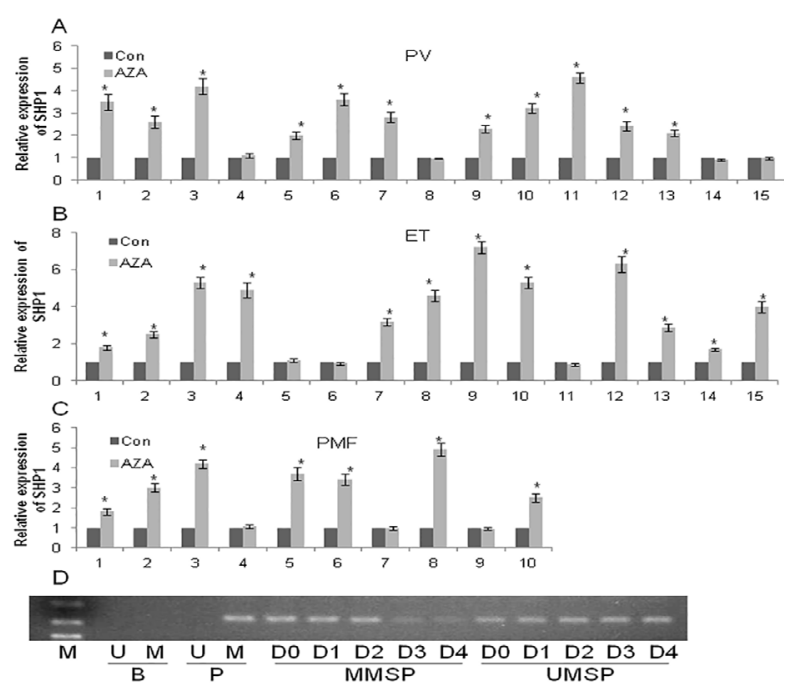

Figure 4. AZA Restores the Expression of SHP1 in Bone Marrow Mononuclear Cells from 15 Patients with PV, 15 with ET, and 10 with PMF. (A-C) Bone marrow mononuclear cells from 15 patients with PV (A), 15 with ET (B), and 10 with PMF (C) were treated with AZA for 4 days, and then the expression of SHP1 was measured by qRT-PCR. $\mathrm{P}<0.01$ verse untreated cells. (D) Representative MMSP and UMSP analysis in bone marrow mononuclear cells treated with AZA from day 0 to day 4

methylation in leukemic cell lines and primary MPN cells. We found methylation of SHP1 frequently occurred in all MPN patients including PV, ET and IMF. Hypermethylation of SHP1 is associated with low expression of SHP1 in leukemic cell lines and primary MPN cells. Treatment with AZA restored the expression of SHP1 by demethylation of SHP1, finally resulting in the inhibition of JAK2/STAT signaling. Taken together, epigenetic inactivation of SHP1 may play an important role in the development of MPN.

As a negative regulator of JAK2/STAT signaling, SHP1 is an attractive target of dysregulation in MPN and leukemia. Although hypermethylation of SHP1 had been reported in leukemia, lymphoma (Oka et al., 2002), colon cancer (Xu et al., 2009) and high-risk myelodysplastic syndrome (Zhang et al., 2012), little data indicated whether SHP-1 was methylated in MPN especially in Chinese MPN patients. Capello et al. (2008) found only fraction of MPN $(8 / 112,7.1 \%)$ showed methylation of SHP-1 using MSP. However, our data suggest SHP1 was methylated in 51/118 (43.2\%) MPN patients. The frequency of methylation in our study is significantly higher than the results from Capello et al. (2008) As reported that MSP may induce the false positive result, bisulfiteconverted DNA from K562 and 10 MPN patients with hypermethylation of SHP1 were performed sequencing. Our data suggest that sequence of CpG island in K562 and 10 MPN patients showed the expected nucleotide change. Thus, our results are reliable. Although Capello et al. reported hypermethylation of SOCS3 occurred in 46 of $112(41.1 \%)$ MPN patients, Fourouclas and his colleage demonstrated hypermethylation of the SOCS3 promoter was only identified in 16 of $50(32 \%)$ patients with IMF but not in patients with ET and PV (Capello et al., 2008). We speculate that the reason for this discrepancy may be the different selection of $\mathrm{CpG}$ island prediction methods, different PCR specificity and sensitivity, or a different patient selection criteria. Otherwise, it is possible that the status of SHP1 methylation in Chinese MPN patients is different from that in Western MPN patients. Several reports pointed out the different clinical and laboratory features in Chinese MPN patients compared with Western MPN patients. Xu et al. (2012) compared clinical and laboratory features of 642 consecutive IMF patients in China and Western MPN patients. They found Chinese patients were significantly younger, fewer had constitutional symptoms, and fewer had a palpable spleen or liver. Furthermore, survival of Chinese IMF patients was significantly longer than that of western populations (Xu et al., 2012). They further found JAK2 46/1 genotype may not explain the different clinical phenotypes of Chinese and Western patients with PMF and other MPN patients. Other mechanisms, presumably genetic or epigenetic, might account for the difference (Wang et al., 2013). Therefore, more studies are required to determine the difference of SHP1 methylation in Chinese populations and Western populations.

Constitutive activation of JAK2/STAT signaling, which is mainly caused by JAK2V617F mutation, has been considered as the major pathogenesis of MPN. SOCS and SHP protein families negatively regulate JAK2/ STAT signaling. Inactivation of SHP1 and SOCS $1 / 3$ by epigenetic and other mechanism were frequently found in MPN and leukemia. SOCS3 was inactivated by DNA methylation in more than 40\% MPN (Capello et al., 2008). Moreover, hyperphosphorylaiton of SOCS3 caused by JAK2V617F mutation rendered it unable to inhibit the mutant kinase (Hookham et al., 2007). Inactivation of SHP1 by DNA methylation may enhance the response of tumour cells to cytokines and may explain the wellknown hypersensitivity to haematopoietic growth factors of MPN. Our data showed that SHP1 methylation occurred in MPN patients both with positive JAK2V617F mutation and negative JAK2V617F mutation (42\% verse $45.9 \%$ ). Considering more than $40 \%$ patients with positive JAK2V617F mutation showed SHP1 methylation, epigenetic inactivation of SHP1 may cooperate with JAK2V617F mutation to contribute to the pathogenesis of MPN. However, more MPN patients with negative JAK2V617F mutation showed methylation, indicating that methylation of SHP1 might represent a new pathogenetic mechanism alternative to JAK2V617F mutation.

As constitutive activation of JAK2/STAT signaling is the main pathogenesis of MPN, inhibition of JAK2/STAT signaling by JAK2 kinase inhibitors has been widely researched (Tefferi and Vainchenker, 2011). Ruxolitinib had been approved by US Food and Drug Administration (FDA) for the treatment for IMF and had been shown to change the natural history of the disease (Verstovsek et al., 2012a). However, most of JAK2 inhibitors showed limited efficacy in the clinical test and did not change the natural history of MPN. Considering the limitations of singleagent JAK2 inhibitor therapy, JAK2-based combinatorial approaches are being developed. Several preclinical studies indicated combination of JAK2 inhibition and pan-histone deacetylase (HDAC) inhibition improved 
efficacy in MPNs compared with single agent(Wang et al., 2009). Our previous data had showed that HDAC inhibitor sodium butyrate inhibited JAK2/STAT signaling through upregulation of SOCS1 and SOCS3 by triggering the promoter-associated histone acetylation of SOCS1 and SOCS3 in K562 and HEL cell lines (Gao et al., 2013). Our present data indicate that restoration of SHP1 by AZA can inhibit JAK2/STAT signaling in K562 cells. However, whether AZA can regulate the expression of BCR-ABL fusion gene, which leads to the activation of JAK2/STAT signaling in K562 cells, is not determined. We measured the mRNA level of BCR-ABL using qRT-PCR and found no significant difference in AZA-treated K562 cells compared with untreated cells (data not shown). Thus, we conclude that AZA inhibits JAK2/STAT signaling in K562 cells independent of BCR-ABL fusion gene. Therefore, restoration of JAK2/STAT signaling inhibitors (SOCS or SHP1) by epigenetic mechanism may contribute to the clinical efficacy when combination of JAK2 inhibitors or HDAC inhibitors.

Several transcription factors, such as Sp1, Oct-1, PU.1 and NF-KB, regulate the expression of SHP-1 through binding SHP1 P2 promoter region (Khoury et al., 2004; Nakase et al., 2009; Kumagai et al., 2012). Because many of these $\mathrm{CpG}$ sites are either adjacent or overlapping these transcriptional elements, increased methylation may block transcription factor binding and consequently interfere with transcriptional activation of the SHP-1 gene (Kumagai et al., 2012), finally leading to the inactivation of SHP1. Zhang et al. (2005) reported that STAT3, DNMT1, and histone deacetylase 1 formed complexes and bound to the SHP-1 promoter in malignant T lymphocytes in vivo. Inhibition of DNMT1 by anti-sense oligonucleotides induced the malignant $\mathrm{T}$ cells DNA demethylation and Reexpression of SHP-1 gene (Zhang et al., 2005). Therefore, treatment with AZA may contribute to the binding of transcript factors to SHP1 promoter P2 and result in the restoration of SHP1 expression owing to demethylation SHP1 promoter.

In conclusion, our results suggest that epigenetic inactivation of SHP1 might be an alternative or complementary mechanism to JAK2 mutations in the pathogenesis of MPD which results in dysregulation of JAK/STAT signal. Restoration of SHP1 expression by AZA-induced demethylation inhibits JAK2/STAT signaling. The identification of promoter hypermethylation affecting components of signaling pathways raises the possibility of the use of demethylating agents as potential therapy in patients with MPN. Combination demethylating agents with JAK2 inhibitors, hydroxyurea, or HDAC inhibitors might be enter into the clinical trials.

\section{Acknowledgements}

This work was supported by National Natural Science Foundation of China (81200350), Zhejiang Provincial Natural Science Foundation of China (LY12H08003; LY13H190006).

\section{References}

Benekli M, Baer MR, Baumann H, et al (2003). Signal transducer and activator of transcription proteins in leukemias. Blood, 101, 2940-54.

Capello D, Deambrogi C, Rossi D, et al (2008). Epigenetic inactivation of suppressors of cytokine signalling in Philadelphia-negative chronic myeloproliferative disorders. Br J Haematol, 141, 504-11.

Chim CS, Fung TK, Cheung WC, et al (2004). SOCS1 and SHP1 hypermethylation in multiple myeloma: implications for epigenetic activation of the Jak/STAT pathway. Blood, 103, 4630-5.

Gao SM, Chen CQ, Wang LY, et al (2013). Histone deacetylases inhibitor sodium butyrate inhibits JAK2/STAT signaling through upregulation of SOCS1 and SOCS3 mediated by HDAC8 inhibition in myeloproliferative neoplasms. Exp Hematol, 41, 261-70.

Hookham MB, Elliott J, Suessmuth Y, et al (2007). The myeloproliferative disorder-associated JAK2 V617F mutant escapes negative regulation by suppressor of cytokine signaling 3. Blood, 109, 4924-9.

James C, Ugo V, Le Couedic JP, et al (2005). A unique clonal JAK2 mutation leading to constitutive signalling causes polycythaemia vera. Nature, $\mathbf{4 3 4}, 1144-8$.

Jost E, do ON, Dahl E, et al (2007). Epigenetic alterations complement mutation of JAK2 tyrosine kinase in patients with BCR/ABL-negative myeloproliferative disorders. Leukemia, 21, 505-10.

Khoury JD, Rassidakis GZ, Medeiros LJ, et al (2004). Methylation of SHP1 gene and loss of SHP1 protein expression are frequent in systemic anaplastic large cell lymphoma. Blood, 104, 1580-1.

Kumagai C, Kalman B, Middleton FA, et al (2012). Increased promoter methylation of the immune regulatory gene SHP-1 in leukocytes of multiple sclerosis subjects. J Neuroimmunol, 246, 51-7.

Levine RL, Wadleigh M, Cools J, et al (2005). Activating mutation in the tyrosine kinase JAK2 in polycythemia vera, essential thrombocythemia, and myeloid metaplasia with myelofibrosis. Cancer Cell, 7, 387-97.

Li LC, Dahiya R (2002). MethPrimer: designing primers for methylation PCRs. Bioinformatics, 18, 1427-31.

Nakase K, Cheng J, Zhu Q, et al (2009). Mechanisms of SHP$1 \mathrm{P} 2$ promoter regulation in hematopoietic cells and its silencing in HTLV-1-transformed T cells. J Leukoc Biol, 85, 165-74.

Oh ST, Simonds EF, Jones C, et al (2010). Novel mutations in the inhibitory adaptor protein LNK drive JAK-STAT signaling in patients with myeloproliferative neoplasms. Blood, 116, 988-92.

Oka T, Ouchida M, Koyama M, et al (2002). Gene silencing of the tyrosine phosphatase SHP1 gene by aberrant methylation in leukemias/lymphomas. Cancer Res, 62, 6390-4.

Pardanani A, Gotlib JR, Jamieson C, et al (2011). Safety and efficacy of TG101348, a selective JAK2 inhibitor, in myelofibrosis. J Clin Oncol, 29, 789-96.

Pardanani AD, Levine RL, Lasho T, et al (2006). MPL515 mutations in myeloproliferative and other myeloid disorders: a study of 1182 patients. Blood, 108, 3472-6.

Pietra D, Li S, Brisci A, et al (2008). Somatic mutations of JAK2 exon 12 in patients with JAK2 (V617F)-negative myeloproliferative disorders. Blood, 111, 1686-9.

Shen SH, Bastien L, Posner BI, et al (1991). A protein-tyrosine phosphatase with sequence similarity to the $\mathrm{SH} 2$ domain of the protein-tyrosine kinases. Nature, 352, 736-9.

Tefferi A, Vainchenker W (2011). Myeloproliferative neoplasms: molecular pathophysiology, essential clinical understanding, and treatment strategies. J Clin Oncol, 29, 573-82. 
Tefferi A, Vardiman JW (2008). Classification and diagnosis of myeloproliferative neoplasms: the 2008 World Health Organization criteria and point-of-care diagnostic algorithms. Leukemia, 22, 14-22.

Tyner JW, Bumm TG, Deininger J, et al (2010). CYT387, a novel JAK2 inhibitor, induces hematologic responses and normalizes inflammatory cytokines in murine myeloproliferative neoplasms. Blood, 115, 5232-40.

Verstovsek S, Kantarjian HM, Estrov Z, et al (2012a). Long-term outcomes of 107 patients with myelofibrosis receiving JAK1/ JAK2 inhibitor ruxolitinib: survival advantage in comparison to matched historical controls. Blood, 120, 1202-9.

Verstovsek S, Mesa RA, Gotlib J, et al (2012b). A double-blind, placebo-controlled trial of ruxolitinib for myelofibrosis. $N$ Engl J Med, 366, 799-807.

Wang J, Xu Z, Liu L, et al (2013). JAK2V617F allele burden, JAK2 46/1 haplotype and clinical features of Chinese with myeloproliferative neoplasms. Leukemia, 27, 1763-7

Wang Y, Fiskus W, Chong DG, et al (2009). Cotreatment with panobinostat and JAK2 inhibitor TG101209 attenuates JAK2V617F levels and signaling and exerts synergistic cytotoxic effects against human myeloproliferative neoplastic cells. Blood, 114, 5024-33.

Witkiewicz A, Raghunath P, Wasik A, et al (2007). Loss of SHP-1 tyrosine phosphatase expression correlates with the advanced stages of cutaneous T-cell lymphoma. Hum Pathol, 38, 462-7.

Wlodarski P, Zhang Q, Liu X, et al (2007). PU.1 activates transcription of SHP-1 gene in hematopoietic cells. J Biol Chem, 282, 6316-23.

Xu SB, Liu XH, Li BH, et al (2009). DNA methylation regulates constitutive expression of Stat6 regulatory genes SOCS-1 and SHP-1 in colon cancer cells. J Cancer Res Clin Oncol, 135, 1791-8.

Xu Z, Gale RP, Zhang Y, et al (2012). Unique features of primary myelofibrosis in Chinese. Blood, 119, 2469-73.

Zhang J, Somani AK, Siminovitch KA (2000). Roles of the SHP-1 tyrosine phosphatase in the negative regulation of cell signalling. Semin Immunol, 12, 361-78.

Zhang Q, Wang HY, Marzec M, et al (2005). STAT3- and DNA methyltransferase 1-mediated epigenetic silencing of SHP-1 tyrosine phosphatase tumor suppressor gene in malignant $\mathrm{T}$ lymphocytes. Proc Natl Acad Sci USA, 102, 6948-53.

Zhang Y, Zhao D, Zhao H, et al (2012). Hypermethylation of SHP-1 promoter in patient with high-risk myelodysplastic syndrome and it predicts poor prognosis. Med Oncol, 29, 2359-63. 